\title{
Magnetization dynamics in spin torque nano-oscillators: Vortex state versus uniform state
}

\author{
R. Lehndorff, * D. E. Bürgler, S. Gliga, R. Hertel, P. Grünberg, and C. M. Schneider \\ Institute of Solid State Research, Electronic Properties (IFF-9) and JARA-FIT, Research Center Jülich GmbH, D-52425 Jülich, Germany \\ Z. Celinski \\ Center for Magnetism and Magnetic Nanostructures, University of Colorado at Colorado Springs, \\ Colorado Springs, Colorado 80918, USA \\ (Received 9 February 2009; revised manuscript received 19 May 2009; published 21 August 2009)
}

\begin{abstract}
Current-driven magnetization dynamics in spin torque nano-oscillators (STNOs) is intensely investigated because of its high potential for high-frequency (HF) applications. We experimentally study current-driven HF excitations of STNOs for two fundamental magnetization states of the free layer, namely, vortex state and uniform in-plane magnetization. Our ability to switch between the two states in a given STNO enables a direct comparison of the critical currents, agility, power, and linewidth of the HF output signals. We find that the vortex state has some superior properties, in particular, it maximizes the emitted HF power and shows a wider frequency tuning range at a fixed magnetic field.
\end{abstract}

DOI: $10.1103 /$ PhysRevB.80.054412

PACS number(s): 72.25.Ba, 75.60.Jk, 75.70.Kw, 75.75.+a

The proposal of spin-polarized current-induced magnetization dynamics of Slonczewski ${ }^{1}$ and Berger ${ }^{2}$ initiated a lot of theoretical and experimental studies in the last years. Promising applications have been found in spin-torque magnetic random access memory and spin torque nanooscillators (STNOs). The latter shows a steady precession of the magnetization of the free layer under the action of a spin-polarized dc current. Via the giant magnetoresistance or tunnel magnetoresistance (GMR or TMR) effect this precession generates a high-frequency $(\mathrm{HF})$ voltage oscillation with frequencies in the $\mathrm{GHz}$ range and a rather wide tuning range by dc current and external magnetic field. Still, one drawback of STNOs is their low output power. To achieve useful power levels several groups work on the synchronization of arrays of STNOs. ${ }^{3-5}$ While this is a very promising approach, maximizing the output power of every single STNO is undeniably the first step to do.

There are several possible arrangements for STNOs. Inplane magnetized free and fixed layers with in-plane ${ }^{6}$ or outof-plane external fields, ${ }^{7}$ in-plane magnetized free and perpendicularly magnetized fixed layers, ${ }^{8}$ and free layer magnetized in a vortex state with in-plane magnetized fixed layer ${ }^{9,10}$ have been studied experimentally. Comparing the characteristics - especially output power-of HF excitations of these arrangements from different experiments is not conclusive, because impedance and absolute resistance change, $\Delta R=R_{A P}-R_{P}$, of the samples have a very strong influence on the detected power. Here, we study HF excitations in two of the arrangements mentioned above that we are able to realize in the same sample. While the fixed layer is uniformly inplane magnetized, the free layer is either uniformly in-plane magnetized or in a vortex state. The direct comparison shows some advantages of the vortex state for the application in STNOs.

The samples are fabricated by depositing $150 \mathrm{~nm} \mathrm{Ag/2}$ $\mathrm{nm} \mathrm{Fe} / 6 \mathrm{~nm} \mathrm{Ag/20} \mathrm{nm} \mathrm{Fe/50} \mathrm{nm} \mathrm{Au} \mathrm{by} \mathrm{molecular} \mathrm{beam}$ epitaxy on a cleaned and annealed GaAs(100) substrate. All layers grow epitaxially as is confirmed by in situ low-energy electron diffraction measurements. The Fe layers adopt a bcc structure, which yields a cubic magnetocrystalline aniso- tropy. Bottom electrodes are formed using optical lithography and ion beam etching (IBE). Then the nanopillars are defined by electron beam lithography and IBE. The pillars have a circular cross-section with a diameter of approximately $230 \mathrm{~nm}$. Only the top magnetic layer is laterally confined, while the bottom layer is left extended on the bottom electrode (with a typical width of $15 \mu \mathrm{m}$ ). We control this step by probing the bottom electrodes with magneto-optical Kerr effect measurements. The pillar is then insulated and the sample surface planarized by spinning on a flowable oxide (fOx-12) of approximately $200 \mathrm{~nm}$ thickness and by depositing $50 \mathrm{~nm}$ silicon nitride by plasma-enhanced chemicalvapor deposition. The insulation is removed in small areas above the pillars by IBE and reactive ion beam etching until the pillar tips are uncovered. Finally, Ti 15 nm/Au 200 nm top electrodes are formed by optical lithography, thermal evaporation, and lift-off technique. ${ }^{11}$ Using this process we fabricate nanomagnets of $230 \mathrm{~nm}$ diameter and $20 \mathrm{~nm}$ thickness, which are separated by $6 \mathrm{~nm} \mathrm{Ag}$ from an extended 2-nm-thick Fe layer [see inset in Fig. 1(a)]. The dimensions of the nanomagnets are in a regime where a magnetic vortex structure and a uniform in-plane magnetization are both stable states. ${ }^{12}$ The 2-nm-thick extended layer, on the other hand, will be uniformly magnetized on length scales much larger than the pillar diameter as long as there is at least a small magnetic field suppressing domain formation.

Micromagnetic simulations were performed using a custom-developed finite-element algorithm. In this algorithm, magnetostatic fields are calculated with a combined finite-element/boundary element method as already used in previous studies. ${ }^{13}$ The usual values for Fe material parameters were chosen: saturation magnetization $\mu_{0} M_{S}=2.15 \mathrm{~T}$, cubic anisotropy $K_{c}=48 \mathrm{~kJ} / \mathrm{m}^{3}$, exchange constant $A=2.1$ $\times 10^{11} \mathrm{~J} / \mathrm{m}$, and a value of $\alpha=0.01$ for the Gilbert damping constant.

First we characterize the GMR response by measuring the pillar resistance at $10 \mathrm{~K}$ while sweeping a magnetic field. We apply a dc current of $1 \mathrm{~mA}$ and measure the voltage drop in a four-probe geometry. Figure 1(a) shows a GMR curve, where the magnetic field was aligned in the sample plane and 

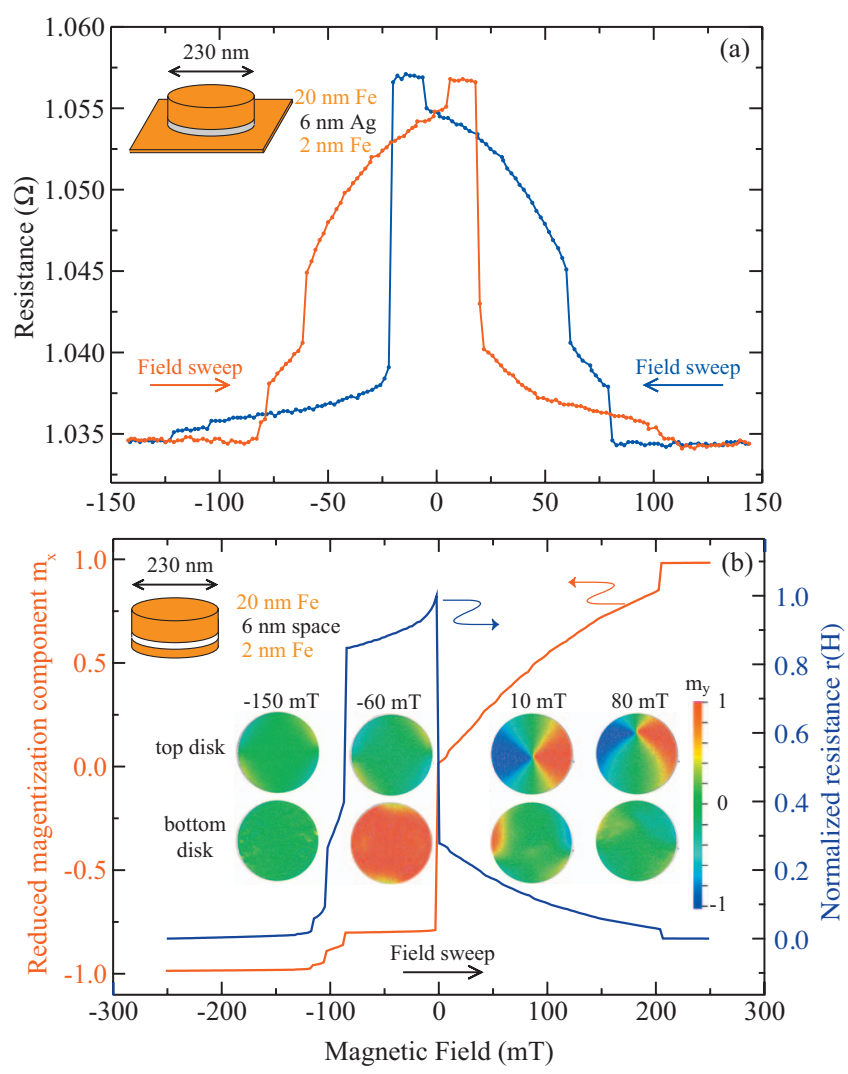

FIG. 1. (Color) (a) Giant magnetoresistance of a pillar structure as sketched in the inset. (b) Micromagnetic simulations of two decoupled disks of $230 \mathrm{~nm}$ diameter and thicknesses of 20 and $2 \mathrm{~nm}$, respectively, separated by a distance of $6 \mathrm{~nm}$ (inset). The pairs of magnetizations patterns show the $m_{y}$ component of the thick (upper) and thin (lower) disks for the indicated fields, which are applied along the magnetic easy $x$ axis. The red curve shows the $x$ component of the reduced magnetization and the blue line the resulting resistance curve. The spike in the resistance at zero field is due to numerical errors in the calculation of the resistance.

parallel to a magnetic easy axis of the Fe layers. Differences to a field sweep parallel to a hard axis are not significant indicating that anisotropy does not play an important role. Starting from saturation at $-150 \mathrm{mT}$ a steep increase in the resistance can be seen at $-70 \mathrm{mT}$. The increase slows down until a plateau of maximum resistance is reached with a small step at a reversed field of $+5 \mathrm{mT}$. At $+20 \mathrm{mT}$ the resistance shows a sudden drop to a level of $1.040 \Omega$ corresponding to a normalized resistance $r(H)=[R(H)$ $\left.-R_{\min }\right] /\left[R_{\max }-R_{\min }\right]$ of 0.25 , where $H$ is the applied field. A slow decrease in the resistance up to $+100 \mathrm{mT}$ is followed by an abrupt decrease to the resistance of the saturated state. The reversed sweep (blue curve) shows a similar behavior.

Micromagnetic simulations of two parallel, decoupled disks of $230 \mathrm{~nm}$ diameter at a vertical distance of $6 \mathrm{~nm}$ and with 20 and $2 \mathrm{~nm}$ thicknesses, respectively, are shown in Fig. 1(b). Based on the comparison to these simulations we can conclude that two distinctly different magnetization states can exist in our samples. The first is dominated by stray field interaction of the thicker layer to the extended layer. While decreasing the magnetic field from saturation [e.g., at $-150 \mathrm{mT}$ in Fig. 1(b)] the magnetization of the two layers undergoes a gradual change from parallel to antiparallel alignment due to stray field interaction $[-60 \mathrm{mT}$ in Fig. 1(b)]. The completely antiparallel alignment is reached at 0 $\mathrm{mT}$ and results in a high resistance. The free layer shows a uniform in-plane magnetization in this field range. The second state is specified by a magnetic vortex in the nanomagnet, which appears after field reversal [+10 mT in Fig. 1(b)]. The formation of the vortex results in a zero-mean in-plane magnetization. The current-perpendicular-plane GMR in Fe/ $\mathrm{Ag} / \mathrm{Fe}$ layers shows a specific angular dependence ${ }^{14,15}$ due to spin accumulation. This results in a drop of the normalized resistance to the experimentally observed value $r \approx 0.25$. Upon further increasing the external field, the vortex core is moved from the center of the disk to the rim $[+80 \mathrm{mT}$ in Fig. 1(b)] until it is expelled at about $+200 \mathrm{mT}$. The simulations qualitatively reproduce the experimental results; quantitative differences of the switching fields most likely arise from reduced magnetization and exchange constant in the nanomagnet as well as from the differences between the simulated and the experimental systems.

In order to keep the computational effort in reasonable bounds, the simulations include only a thin magnetic nanodisk, while in the experiment the thin magnetic layer is extended. Since most of the stray field originates from the thick nanomagnet this approximation should only give minor discrepancies between experiment and simulation. The most obvious difference is that in the simulation the magnetization direction of the thin nanomagnet aligns opposite to the direction of the external field because it has less magnetic moment than the thick nanomagnet. In the experiment, however, it is more likely that the magnetization direction of the thick nanomagnet is turning, since the relevant part of the thin, extended magnetic layer is stabilized by the exchange interaction to the rest of the micron-sized layer. The notable plateau in the measured resistance curve between +5 and $+20 \mathrm{mT}$ is not explained by the micromagnetic simulations. We assume that at $+5 \mathrm{mT}$ the vortex nucleates in the nanodisk and at the same time the reversal of the extended layer happens via domain-wall formation and motion. This may result in a highly nonuniform magnetization state in the extended layer leading to the resistance plateau. At $+20 \mathrm{mT}$ the magnetization in the extended layer is finally reversed and homogeneous yielding a lowered resistance.

Having characterized the behavior of the system in response to externally applied fields, we now address currentinduced switching processes by sweeping the dc current from 0 to $25 \mathrm{~mA}$, then to $-25 \mathrm{~mA}$ and back to $0 \mathrm{~mA}$ at fixed magnetic fields. The initial states were prepared by magnetic field sweeps from positive or negative saturation to the stated field values. Measurements were performed at $10 \mathrm{~K}$. Figure 2 shows the evolution of current-induced switching for different strengths of the magnetic field that was applied parallel to a magnetic easy axis. At 40 and $49 \mathrm{mT}$ full hysteretic switching can be seen (green and red curves), where at positive currents a high-resistive state and at negative currents a low-resistive state is reached. Results depicted in Fig. 1 supports the conclusion that the switching events take place in the nanomagnet rather than the thinner extended reference layer. In our convention, a positive current corresponds to an electron flow from the nanomagnet to the extended layer. In 


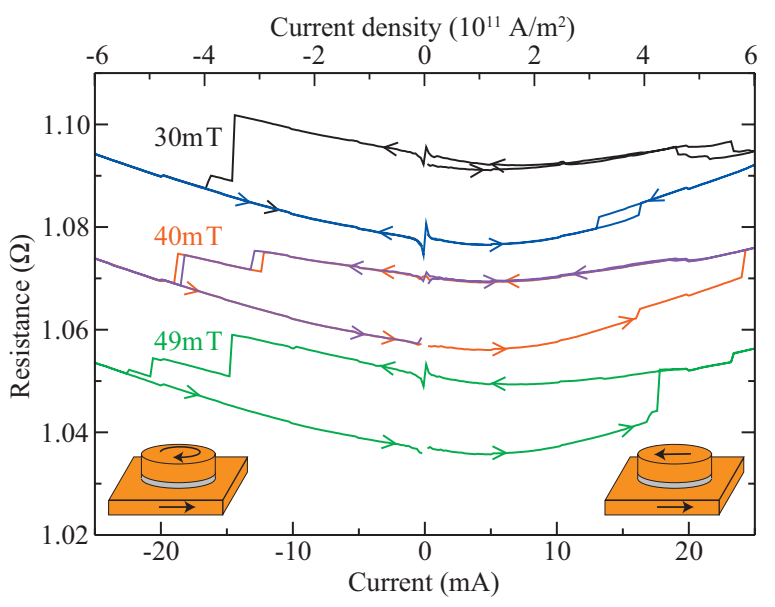

FIG. 2. (Color) Current-induced switching between the lowresistive vortex and high-resistive uniform state. Arrows on the curves indicate the current sweep direction. The black and purple curves start in the uniform state, all others in the vortex state. For clarity the graphs measured at 40 and $30 \mathrm{mT}$ are offset by +20 and $+40 \mathrm{~m} \Omega$, respectively, relative to the green curve.

previous experiments with $\mathrm{Fe} / \mathrm{Ag} / \mathrm{Fe}$ pillars ${ }^{11,15,16}$ we have established that the spin-transfer torque due to a positive current acts toward an antiparallel alignment. Therefore, the high-resistive state can be identified in terms of antiparallel aligned uniform magnetizations and the low-resistive state as the vortex state. The two resistance values near $0 \mathrm{~mA}$ of the unshifted green curve in Fig. 2 measured at $49 \mathrm{mT}$ indeed correspond to the values of the red and blue curves at +49 mT in Fig. 1(a). This interpretation also correlates well with the observed shift of the hysteresis to the left when the magnetic field is increased. The vortex state is less favorable for higher fields than the uniform state. Therefore the switching to the vortex state at negative currents is getting harder. On the other hand, the two graphs recorded at $30 \mathrm{mT}$ (blue and black curves) show that at this weaker field, a switching to the uniform state is no longer possible with currents up to $25 \mathrm{~mA}$ corresponding to $6 \times 10^{11} \mathrm{~A} / \mathrm{m}^{2}$. Simultaneously the magnitude of the critical current for switching from the uniform state to the vortex state is decreasing. For the black curve at $30 \mathrm{mT}$ and the purple one at $40 \mathrm{mT}$ the measurement started from the uniform state. The fact that we do not observe a switching to the vortex state at positive currents in these measurements shows that the prevalent torque in the switching processes does not originate from Oersted fields. These circumferential fields tend to switch the magnetization of the nanomagnet into a vortex state also at positive currents, just with the opposite vorticity compared to negative currents.

We measure dc current-induced HF excitations of the magnetization at room temperature by amplification and detection of voltage oscillations across the nanopillar using a microwave probe station. The voltage variation arises from the GMR of the $\mathrm{Fe} / \mathrm{Ag} / \mathrm{Fe}$ stack, which reaches $2 \%$ or $22 \mathrm{~m} \Omega$ in Fig. 1(a). The impedance of our sample was $11 \Omega$ at $1.5 \mathrm{GHz}$. Figure 3(a) shows the HF response of a nanopillar with magnetization in the uniform state measured in an external field of $82 \mathrm{mT}$ applied along an easy axis of the $\mathrm{Fe}$
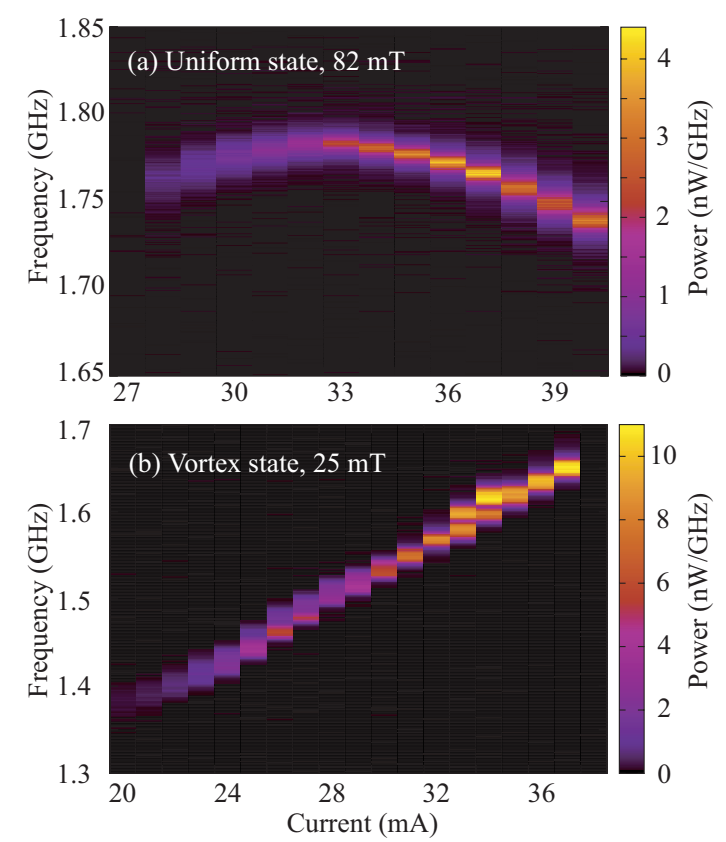

FIG. 3. (Color) Spin-transfer torque induced excitation of qualitatively different oscillatory modes in a nanodisk. (a) After preparation of a uniform state a standing-wave mode with a transition from blueshift to redshift is excited; measurement at $82 \mathrm{mT}$. (b) The gyrotropic mode is excited after preparation of the vortex state; measurement at $25 \mathrm{mT}$. Note that the microwave output power generated by the gyrating vortex for a given dc current in (b) is much higher than for the standing-wave mode in (a).

layers. The observed behavior is qualitatively similar for other field values. We are able to detect this excitation between 35 and $82 \mathrm{mT}$ with maximum frequencies only slightly increasing from 1.67 to $1.78 \mathrm{GHz}$. In the shown case, at a threshold current of $27 \mathrm{~mA}$ a weak signal appears at $1.762 \mathrm{GHz}$. The amplitude and frequency increase slightly with increasing current up to $1.783 \mathrm{GHz}$ at $32 \mathrm{~mA}$, i.e., the observed mode is blueshifting in this current range. At 32 $\mathrm{mA}$ the peak width reaches a minimum of $8 \mathrm{MHz}$. For further increasing current the frequency redshifts to $1.739 \mathrm{GHz}$ at the maximum applied current of $40 \mathrm{~mA}$ while the peak broadens to $11 \mathrm{MHz}$. The mode agility in this regime is about $-6 \mathrm{MHz} / \mathrm{mA}$. A maximum power of $3.2 \mathrm{pW} /\left(\mathrm{GHz} \mathrm{mA}^{2}\right)$ is reached at a current of $36 \mathrm{~mA}$.

The low frequencies of the excitations are a result of the cancellation of the dipolar coupling field of about $80 \mathrm{mT}$ by the external field. We assume a standing-wave mode, where the rather large size of the element leads to low-frequency excitations, too. The observed blueshift behavior at low currents can be interpreted in terms of such a standing-wave mode, as well. ${ }^{17}$ At higher currents the redshift sets in which is explained by a predominantly homogeneous in-plane precession of the magnetization. For increasing current the angle of precession increases, thus leading to a lower frequency. ${ }^{18}$ At the same time the peak width increases.

Figure 3(b) shows representative HF excitations of a nanopillar with magnetization in the vortex state measured at an external in-plane field of $25 \mathrm{mT}$. At positive currents between 20 and $37 \mathrm{~mA}$ a signal with frequency increasing lin- 
TABLE I. Comparison of most relevant characteristics of the HF excitations of uniform and vortex states. The minimal critical current for the uniform state is $19 \mathrm{~mA}$ at an in-plane magnetic field of $48 \mathrm{mT}$.

\begin{tabular}{|c|c|c|}
\hline Free layer in: & Uniform state & Vortex state \\
\hline Min. Critical current density $\left(10^{11} \mathrm{~A} / \mathrm{m}^{2}\right)$ & 4.6 & 4.8 \\
\hline Agility (MHz/mA) & -6 & 17 \\
\hline Spanned freq. range $(\mathrm{MHz})$ & 44 & 270 \\
\hline Max. power $\left[\mathrm{pW} /\left(\mathrm{GHz} \times \mathrm{mA}^{2}\right)\right]$ & 3.2 & 9.3 \\
\hline Min. linewidth (MHz) & 8 & 14 \\
\hline
\end{tabular}

early from 1.38 to $1.65 \mathrm{GHz}$ is measured. The resulting mode agility is $+17 \mathrm{MHz} / \mathrm{mA}$. The smallest linewidth of 14 $\mathrm{MHz}$ is achieved at $32 \mathrm{~mA}$. The maximum power of $9.3 \mathrm{pW} /\left(\mathrm{GHz} \mathrm{mA}^{2}\right)$ is reached at a current of $34 \mathrm{~mA}$. At some values of the current two peaks are present, thus showing a mode-hopping behavior. This vortex excitation is only present in small fields from 10 up to $42 \mathrm{mT}$ and shows a frequency increase from 1.52 to $1.72 \mathrm{GHz}$ with increasing field. A comparison of the most relevant characteristics for the two states is compiled in Table I.

Here, the gyrotropic mode ${ }^{19}$ of the vortex is excited as previously reported by Pribiag et al. ${ }^{9}$ There is a significant difference in the HF excitations observed for the same nanopillar with magnetization in the uniform state. This difference is caused by the strongly inhomogeneous magnetization pattern of the magnetic vortex. The current exerts spintransfer torque on the vortex in such a way that the vortex core is moved from its equilibrium position in the center of the nanodisk, but the internal structure of the vortex does not change. As a result the vortex starts a circular motion around the equilibrium position. If a steady spin-transfer torque excitation acts on the vortex, it will find a stable elliptic or circular trajectory with a radius proportional to the excitation amplitude. When for increasing current the trajectory approaches the rim of the disk, the vortex experiences a stronger restoring force, increasing its precessional frequency. This results in a linear increase in the frequency yielding a linear mode agility of $+17 \mathrm{MHz} / \mathrm{mA}$ [Fig. 3(b)]. An inplane magnetic field will move the center of the vortex gy- ration to the rim of the disk, thus also leading to an increase in frequency. This dynamic mode is described by the movement of a rigid magnetic structure or particle rather than by a standing-wave pattern as is the case for the uniform state. At each spot within the trajectory of the vortex core, the magnetization rotates during one period of the gyrotropic cycle by full $2 \pi$ about the sample normal. Thus, for a vortex core moving on a trajectory close to the rim of the sample the product of oscillation amplitude times area, where oscillations take place, is maximized. As a consequence, the emitted power of the nanopillar in the vortex state is nearly three times the power emitted in the uniform state (Fig. 3 and Table I).

We studied HF excitations of a vortex and a uniform state in the same sample. Due to negligible variation in the sample impedance over the explored frequency range, we can directly compare the output power and other properties of the excitations. Higher agility, wider tuning range, and higher output power are all advantageous for the application of the vortex state in STNOs, while a lower linewidth is achieved in the uniform state. Although this conclusion is derived from metallic, GMR-type STNOs, our generic, micromagnetic arguments make it valid also for TMR-based STNOs, which intrinsically generate higher output power. ${ }^{20}$

We would like to thank R. Schreiber and F.-J. Köhne for technical support. This work was partly funded by the German academic exchange service (DAAD) while at UCCS by the NSF (Grant No. DMR-0605629) and the ARO (Grant No. W911NF-04-1-0247).

\footnotetext{
*Present address: Bielefeld University, D-33615 Bielefeld, Germany; rlehn@physik.uni-bielefeld.de

${ }^{1}$ J. Slonczewski, J. Magn. Magn. Mater. 159, L1 (1996).

${ }^{2}$ L. Berger, Phys. Rev. B 54, 9353 (1996).

${ }^{3}$ S. Kaka, M. R. Pufall, W. H. Rippard, T. J. Silva, S. E. Russek, and J. A. Katine, Nature (London) 437, 389 (2005).

${ }^{4}$ F. B. Mancoff, N. D. Rizzo, B. N. Engel, and S. Tehrani, Nature (London) 437, 393 (2005).

${ }^{5}$ J. Grollier, V. Cros, and A. Fert, Phys. Rev. B 73, 060409(R) (2006).

${ }^{6}$ S. I. Kiselev, J. C. Sankey, I. N. Krivorotov, N. C. Emley, R. J. Schoelkopf, R. A. Buhrman, and D. C. Ralph, Nature (London) 425, 380 (2003).

${ }^{7}$ W. H. Rippard, M. R. Pufall, S. Kaka, T. J. Silva, and S. E.
}

Russek, Phys. Rev. B 70, 100406(R) (2004).

${ }^{8}$ D. Houssameddine, U. Ebels, B. Delaët, B. Rodmacq, I. Firastrau, F. Ponthenier, M. Brunet, C. Thirion, J.-P. Michel, L. Prejbeanu-Buda, M.-C. Cyrille, O. Redon, and B. Dieny, Nature Mater. 6, 447 (2007)

${ }^{9}$ V. S. Pribiag, I. N. Krivorotov, G. D. Fuchs, P. M. Braganca, O. Ozatay, J. C. Sankey, D. C. Ralph, and R. A. Buhrman, Nat. Phys. 3, 498 (2007).

${ }^{10}$ Q. Mistral, M. van Kampen, G. Hrkac, J.-V. Kim, T. Devolder, P. Crozat, C. Chappert, L. Lagae, and T. Schrefl, Phys. Rev. Lett. 100, 257201 (2008).

${ }^{11}$ H. Dassow, R. Lehndorff, D. E. Bürgler, M. Buchmeier, P. A. Grünberg, C. M. Schneider, and A. van der Hart, Appl. Phys. Lett. 89, 222511 (2006). 
${ }^{12}$ R. P. Cowburn, J. Phys. D 33, R1 (2000).

${ }^{13}$ R. Hertel, O. Fruchart, S. Cherifi, P. O. Jubert, S. Heun, A. Locatelli, and J. Kirschner, Phys. Rev. B 72, 214409 (2005).

${ }^{14}$ J. Slonczewski, J. Magn. Magn. Mater. 247, 324 (2002).

${ }^{15}$ R. Lehndorff, M. Buchmeier, D. E. Bürgler, A. Kakay, R. Hertel, and C. M. Schneider, Phys. Rev. B 76, 214420 (2007).

${ }^{16}$ R. Lehndorff, D. E. Bürgler, A. Kakay, R. Hertel, and C. M. Schneider, IEEE Trans. Magn. 44, 1951 (2008).
${ }^{17}$ A. Kákay, S. Gliga, R. Hertel, R. Lehndorff, D. E. Bürgler, and C. M. Schneider (unpublished).

${ }^{18}$ A. N. Slavin and P. Kabos, IEEE Trans. Magn. 41, 1264 (2005).

${ }^{19}$ K. Y. Guslienko, B. A. Ivanov, V. Novosad, Y. Otani, H. Shima, and K. Fukamichi, J. Appl. Phys. 91, 8037 (2002).

${ }^{20}$ A. M. Deac, A. Fukushima, H. Kubota, H. Maehara, Y. Suzuki, S. Yuasa, Y. Nagamine, K. Tsunekawa, D. D. Djayaprawira, and N. Watanabe, Nat. Phys. 4, 803 (2008). 